\title{
ISOLATION, MOLECULAR IDENTIFICATION AND APPLICATION OF EXOGENOUS INDOLE ACETIC ACID (IAA) PRODUCING PLANT GROWTH PROMOTING RHIZOBACTERIA (PGPR) FROM SOIL
}

\author{
ABDUL JALEEL.R ${ }^{1}$, JADHEER AHSAN K.P ${ }^{2}$, SWAGATO BHATTACHARJEE ${ }^{3}$, \\ S. MOHANA PRIYA ${ }^{4}$, Dr.PRAVEENA ${ }^{5}$ \\ ${ }^{1,2,5}$ Mohammed Sathak Arts and Science College, University Of Madras, Tamil Nadu \\ ${ }^{3}$ DBT-Rajiv Gandhi Centre for Biotechnology (RGCB) \\ ${ }^{4}$ PSG College of Arts and Science, Coimbatore.
}

\begin{abstract}
Plant growth promoting rhizobacteria (PGPR) are often used as inoculants to promote the growth and yield of agricultural crops, however PGPR strain selection is extremely important. This bacteria colonies plant roots and promote plant growth through a variety of mechanisms including phytohormone production, improved water and nutrient uptake, improved nitrogen availability in the soil, production of ACC-deaminase for ethylene breakdown, phosphate solubilization, siderophore production, phytoremdiation, and phytopathogen defense. Microbial Auxin production is the primary component that promotes healthy plant growth and development. The ability of PGPR to produce phytohormones can be used to boost plant growth, reducing the need for chemical fertilizers and their harmful effects on the environment. The work in this study includes isolation, screening, molecular identification, and applying effective PGPR strains based on their ability to produce auxin in vitro. The soil samples were serially diluted, and the appropriate dilution was placed onto nutrient agar media. Invitro screening was performed on isolated colonies using the Salkowski reagent, which results in a pink coloring. The existence of IAA producers is indicated by the appearance of pink colour. This isolated organism was subjected to DNA isolation after passing morphological and biochemical tests. 16S PCR was used to amplify the isolated DNA. The bacteria was identified as Pseudomonas fluorescence after sequencing and analysing the amplified products with BLAST. When compared to other strains, Pseudomonas has a higher ability to produce auxin. In addition, a pot experiment in plants was carried out to test the influence of auxin (IAA) production by the isolated strain.
\end{abstract}

Keywords : PGPR, Indole acetic acid,BLAST

1) INTRODUCTION

Crop yields must be increased urgently in order to meet the demands of providing food to the world's ever-increasing population. The use of chemical fertilizers and pesticides for diverse purposes has increased in tandem with the rate of population growth Although chemical fertilizers and pesticides produce good results, their disadvantages, such as pollution of vast water resources, loss of microorganisms, soil acidity, and reduction in soil fertility, are currently jeopardizing agricultural processes (Khin, 2012). In recent years, scientists have moved their attention to the potential of beneficial microbes, and the use of plant growth promoting rhizobacteria (PGPR) for sustainable agriculture has expanded in many parts of the world.

Isolation and identification of exogenous IAA producing plant growth promoting rhizobacteria (PGPR) from agriculture soil and their application.

Sample collection

At Kottakkal, Malappuram, soil samples were taken from the rhizosphere of the Mimosa pudica (Touch me not or thottavadi) plant. The intact root system was scraped out, and rhizospheric soil samples were carefully gathered in plastic 
International Advanced Research Journal in Science, Engineering and Technology

Vol. 8, Issue 10, October 2021

DOI: $10.17148 /$ IARJSET.2021.81007

bags and used for rhizosphere PGPR isolation.

Isolation of organism using specific media

Subjected to serial dilution .For this, Two dilution test tubes $\left(10^{-2}\right.$ and $\left.10^{-3}\right)$ were selected and performing spread plate.

$>\quad$ Screening for IAA

Quantitative \& Qualitative Assay

$>\quad$ Microscopical and Biochemical identification of bacteria

$>\quad$ Molecular identification

Cell lysis, deactivation of cellular nucleases, and separation of the desired nucleic acids from cellular detritus are all required for the extraction and purification of DNA from biological material. Mechanical disruption, chemical treatment, and enzymatic digestion are all common lysis procedures.

3) RESULTS AND DISCUSSION

\subsection{Quantitative Assay}

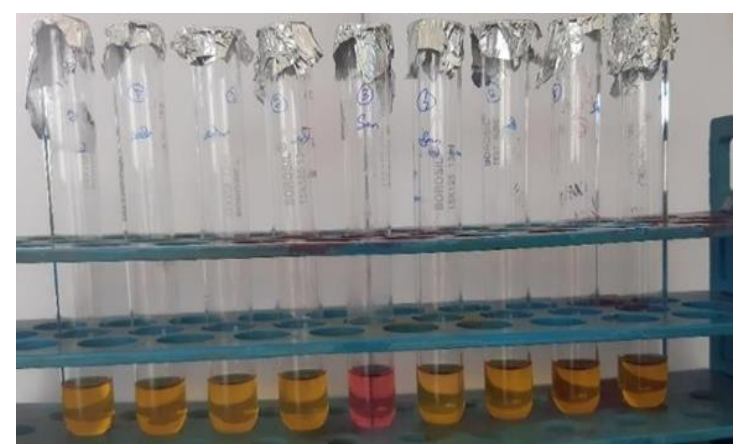

Fig1: The pink colour shows IAA producing culture without tryptophan

\subsection{Pure Culturing Of Selected PGPR}

\subsection{Gram's Staining}

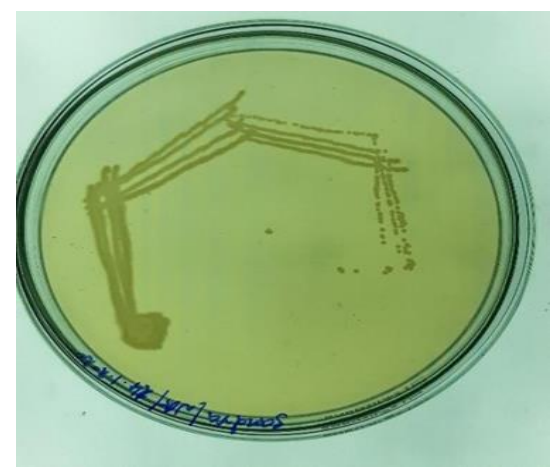

Fig 2: Quadrant streak of isolated colony

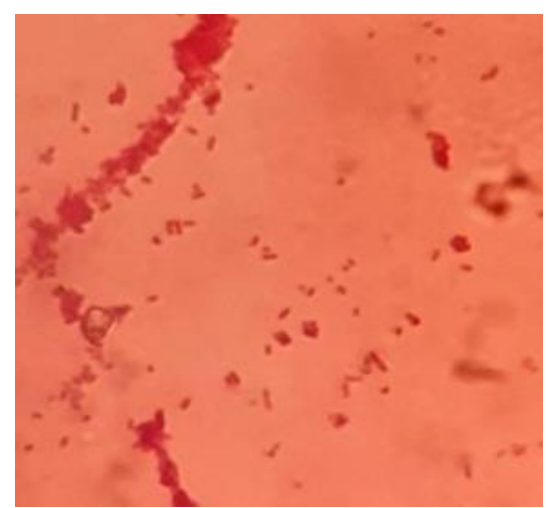

Fig3 : Gram negative rod shaped bacteria 
International Advanced Research Journal in Science, Engineering and Technology

Vol. 8, Issue 10, October 2021

\subsection{Biochemical Test}

DOI: $10.17148 /$ IARJSET.2021.81007

\begin{tabular}{|l|l|} 
TEST & RESULT \\
\hline INDOLE & Negative \\
\hline MR & Positive \\
\hline VP & Negative \\
\hline Urease & Positive \\
\hline OXIDASE & Positive \\
\hline CATALASE & Positive \\
\hline \hline CITRATE & Positive \\
\hline
\end{tabular}

Fig.4Biochemical Test Result

3.5 Isolation Of Bacterial Genomic DNA

Agarose Gell Electrophoresis (AGE)

\begin{tabular}{|l|l|}
\hline LANE 1 & $\begin{array}{l}\text { Reference } \\
\text { DNA }\end{array}$ \\
\hline LANE 2 & $\begin{array}{l}\text { Isolated } \\
\text { DNA }\end{array}$ \\
\hline
\end{tabular}

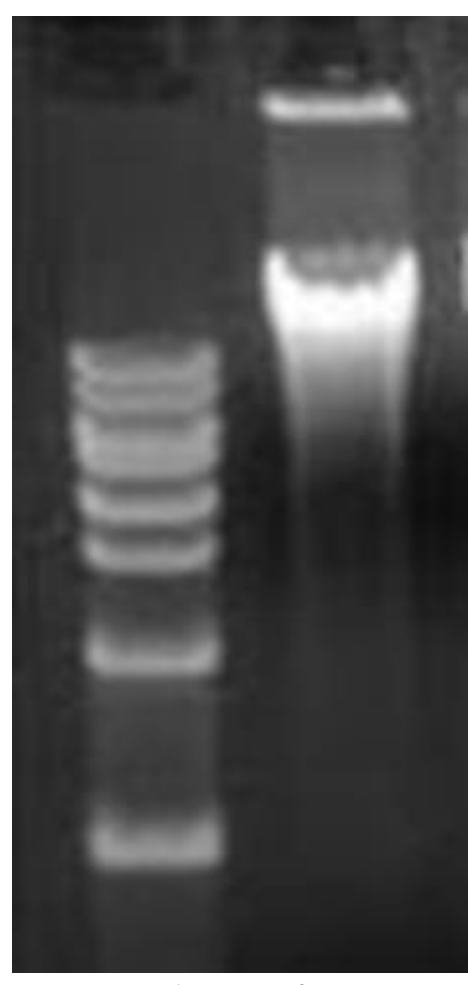

12

Fig5 :Bands observed under UV illumination

Amplification Using 16S PCR

\begin{tabular}{|l|c|}
\hline LANE 1 & 100bp MARKER \\
\hline LANE 2 & 16 S GENE PCR P \\
\hline
\end{tabular}


Vol. 8, Issue 10, October 2021

DOI: 10.17148/IARJSET.2021.81007

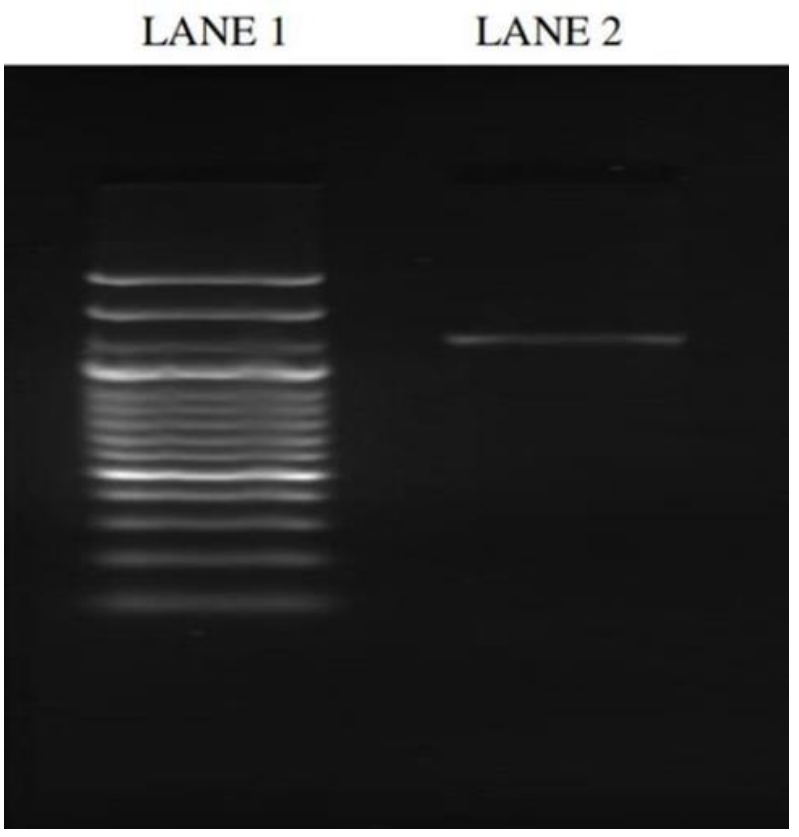

Fig6: Agarose Gel Electrophoresis of 16s ribosomal RNA gene

\section{$>\quad$ BLAST}

Blast was done and $99.88 \%$ similarity was noted with 16s rRNA gene of the organismPseudomonas fluorescens.

\begin{tabular}{|c|c|c|c|c|c|c|c|}
\hline & Description & $\begin{array}{l}\text { Max } \\
\text { Score }\end{array}$ & $\begin{array}{l}\text { Total } \\
\text { Score }\end{array}$ & $\begin{array}{l}\text { Query } \\
\text { Cover }\end{array}$ & $\begin{array}{c}E \\
\text { value }\end{array}$ & $\begin{array}{l}\text { Per. } \\
\text { Ident }\end{array}$ & Accession \\
\hline - & Pseudomonas fluorescens strain FP23 16 S ribosomal RNA gene,..F & 1580 & 1580 & $100 \%$ & 0.0 & $99.88 \%$ & $\underline{\mathrm{DQ} 2}$ \\
\hline 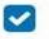 & Pseudomonas sp. strain Fas $1416 \mathrm{~S}$ ribosomal RNA gene, partial st & 1574 & 1574 & $100 \%$ & 0.0 & $99.77 \%$ & MH235971.1 \\
\hline 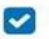 & Pseudomonas fluorescens strain VNS01 $16 \mathrm{~S}$ ribosomal RNA gene & 1574 & 1574 & $99 \%$ & 0.0 & $99.88 \%$ & KF758403.1 \\
\hline 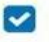 & Pseudomonas fluorescens partial 16S rRNA gene, isolate s4 & 1570 & 1570 & $100 \%$ & 0.0 & $99.65 \%$ & HF913576.1 \\
\hline 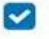 & Pseudomonas azotoformans partial $16 \mathrm{~S}$ rRNA gene, isolate SW_H & 1568 & 1568 & $100 \%$ & 0.0 & $99.65 \%$ & $\underline{\text { LR722854.1 }}$ \\
\hline$\checkmark$ & Pseudomonas sp. strain RGM_2656 16S ribosomal RNA gene,par & 1568 & 1568 & $100 \%$ & 0.0 & $99.65 \%$ & MN786797.1 \\
\hline$\checkmark$ & Pseudomonas gessardii strain OBE3 16 S ribosomal RNA gene,.par & 1568 & 1568 & $100 \%$ & 0.0 & $99.65 \%$ & MN685265.1 \\
\hline$\checkmark$ & Pseudomonas fluorescens strain ORTB3 $16 \mathrm{~S}$ ribosomal RNA gene & 1568 & 1568 & $100 \%$ & 0.0 & $99.65 \%$ & MN685247.1 \\
\hline 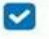 & Pseudomonas sp. strain 1JPC $16 \mathrm{~S}$ ribosomal RNA gene, partial se & 1568 & 1568 & $100 \%$ & 0.0 & $99.65 \%$ & MN651328.1 \\
\hline ح & Pseudomonas sp. CFSAN084952 chromosome, complete genome & 1568 & 9413 & $100 \%$ & 0.0 & $5 \%$ & CP045767.1 \\
\hline$\checkmark$ & Pseudomonas azotoformans strain R-58 $16 \mathrm{~S}$ ribosomal RNA gene & 1568 & 1568 & $100 \%$ & 0.0 & $99.65 \%$ & $\underline{\text { MN5 }}$ \\
\hline$\checkmark$ & Pseudomonas paralactis strain CFH1-01 16S ribosomal RNA gene & 1568 & 1568 & $100 \%$ & 0.0 & $99.65 \%$ & MN559438.1 \\
\hline 5 & Pseudomonas costantinii strain P4M150 16S ribosomal RNA gene & 1568 & 1568 & $100 \%$ & 0.0 & $99.65 \%$ & MN421425.1 \\
\hline$\checkmark$ & Pseudomonas costantinii strain P4M97 16S ribosomal RNA gene. & 1568 & 1568 & $100 \%$ & 0.0 & $99.65 \%$ & MN421413.1 \\
\hline$\checkmark$ & Pseudomonas costantinii strain P4M92 16S ribosomal RNA gene. & 1568 & 1568 & $100 \%$ & 0.0 & $99.65 \%$ & MN421412.1 \\
\hline ح & Pseudomonas costantinii strain P4M90 16 S ribosomal RNA gene, & 1568 & 1568 & $100 \%$ & 0.0 & $99.65 \%$ & MN421411. \\
\hline
\end{tabular}

Fig 7: Blast result

\section{4) CONCLUSION}

The results of this investigation clearly showed that the isolate tested could make IAA and, as a result, was classified as an IAA-producing rhizobacterium. P.fluorescens was the most recently isolated organism. This has the ability to create IAA, which was investigated on plants as part of this research. When compared to other species, Pseudomonas species are capable of creating more IAA. This research is important because it suggests that using PGPR as inoculants or bio fertilisers is an effective way to replace chemical fertilisers. 


\title{
International Advanced Research Journal in Science, Engineering and Technology
}

\author{
Vol. 8, Issue 10, October 2021
}

\section{DOI: $10.17148 /$ IARJSET.2021.81007 \\ 5) REFERENCES}

I. Ashrafuzzaman, M., Hossen, F. A., Ismail, M. R., Hoque, A., Islam, M. Z., Shahidullah, S. M., \& Meon, S. (2009). Efficiency of plant growthpromoting rhizobacteria (PGPR) for the enhancement of rice growth. African Journal of Biotechnology, 8(7).

II. Bianciotto, V., Andreotti, S., Balestrini, R., Bonfante, P., \& Perotto, S. (2001). Mucoidmutants of the biocontrol strain Pseudomonas fluorescens CHA0 show increased abilityin biofilm formation on mycorrhizal and nonmycorrhizal carrot roots. Molecular plant-microbe interactions, 14(2), 255-260

III. Cassana, J. (2009). The breakfast cereal survey: teaching archaeology with 12 essentialvitamins and minerals. SAA archaeological record, 9(4), 1318.

IV. Farzana, Y., \& Radizah, O. (2005). Influence of rhizobacterial inoculation on growth of the sweet potato cultivar. OnLine Journal of Biological Science, 1(3), 176-179.

V. Glick, B. R. (2012). Plant growth-promoting bacteria: mechanisms andapplications. Scientifica, 2012.

VI. Khan, M. S., Zaidi, A., Wani, P. A., \& Oves, M. (2009). Role of plant growth promotingrhizobacteria in the remediation of metal contaminated soils. Environmental chemistryletters, 7(1), 1-19.

VII. Lifshitz, R., Kloepper, J. W., Kozlowski, M., Simonson, C., Carlson, J., Tipping, E. M.,\& Zaleska, I. (1987). Growth promotion of canola (rapeseed) seedlings by a strain of Pseudomonas putida under gnotobiotic conditions. Canadian Journal of Microbiology, 33(5), 390-395.

VIII. Muleta, D., Assefa, F., Börjesson, E., \& Granhall, U. (2013). Phosphate-solubilising rhizobacteria associated with Coffea arabica L. in natural coffee forests of southwesternEthiopia. Journal of the Saudi Society of Agricultural Sciences, 12(1), 73-84.

IX. Moeinzadeh, A., Sharif-Zadeh, F., Ahmadzadeh, M., \& Tajabadi, F. (2010). Biopriming of Sunflower ('Helianthus annuus' L.) Seed with'Pseudomonas fluorescens'for Improvement of Seed Invigoration and Seedling Growth. Australian Journal of Crop Science, 4(7), 564

X. Patel, K., Goswami, D., Dhandhukia, P., \& Thakker, J. (2015). Techniques to study microbial phytohormones. In Bacterial metabolites in sustainable agroecosystem (pp. 1-27). Springer, Cham.

XI. Sivasakthi, S., Usharani, G., \& Saranraj, P. (2014). Biocontrol potentiality of plant growth promoting bacteria (PGPR)-Pseudomonas fluorescens and Bacillus subtilis: A review. African journal of agricultural research, 9(16), 1265-1277.

XII. Somers, E., Vanderleyden, J., \& Srinivasan, M. (2004). Rhizosphere bacterial signalling: a love parade beneath our feet. Critical reviews in microbiology, 30(4), 205-240.

XIII. Sudha, M., Gowri, R. S., Prabhavathi, P., Astapriya, P., Devi, S. Y., \& Saranya, A. (2012). Production and optimization of indole acetic acid by indigenous micro flora using agro waste as substrate. Pakistan Journal of Biological Sciences, 15(1), 39.

XIV. Touceda-González, M., Brader, G., Antonielli, L., Ravindran, V. B., Waldner, G., Friesl-Hanl, W., ... \& Sessitsch, A. (2015). Combined amendment of immobilizersand the plant growth-promoting strain Burkholderia phytofirmans PsJN favours plant growth and reduces heavy metal uptake. Soil Biology and Biochemistry, 91, 140-150. 